\title{
A Study of the Germination of Green Beans in Different Gravity Conditions
}

\author{
Joompon Bamrungwong, Bancha Arthibenyakul
}

Department of Industrial Physics and Medical Instrumentation, Faculty of Applied Science, King Mongkut's University of Technology North Bangkok, Bangkok, Thailand

Email address:

joompondej@gmail.com (J. Bamrungwong),bancha.a@sci.kmutnb.ac.th (B. Arthibenyakul)

\section{To cite this article:}

Joompon Bamrungwong, Bancha Arthibenyakul. A Study of the Germination of Green Beans in Different Gravity Conditions. World Journal of Applied Physics. Vol. 4, No. 1, 2019, pp. 12-16. doi: 10.11648/j.wjap.20190401.12

Received: March 5, 2019; Accepted: April 13, 2019; Published: June 4, 2019

\begin{abstract}
The literature suggests that plants are able to grow in the absence of gravity, and NASA has a plan to cultivate plants on Mars. Thus, the aim of this research was to study the germination and growth of green beans at differing levels of gravity from $0.5 \mathrm{G}$ to $3 \mathrm{G}$. Pseudo gravity has been used to study the growth and germination of the green beans under different gravity levels. The results showed that under high gravity the green beans were able to germinate but were unable to grow. Under low gravity the green beans were able to both germinate and grow. However, due to the limitations of the authors' apparatus, the behavior of green beans under lower gravity levels than $0.5 \mathrm{G}$ could not be investigated. Finite element modeling was used to study the stems of the green beans at different gravity levels. The result from the FEM is correlated with the experiment but it did not take the angle from the horizontal line as the actual germination of the green beans. However, it shows that under high gravity the stems of the bean sprouts were not able to resist the force of gravity. Thus, the plants might be unable to germinate and grow on new planets of larger size.
\end{abstract}

Keywords: Green Beans, Growth, Germination, Gravity

\section{Introduction}

There was a research study which stated that plants and vegetables were able to grow in a Space Garden $[1,9]$. The experiment was conducted to investigate the possibility of growing plants on Mars or the moon, and used soils simulating those environments. The results show that plants were able to germinate and grow in both Martian and Lunar soils for a period of 50 days without the addition of any nutrients. The plant used in this research is the green bean (Mung bean). The green bean seeds which have geminated are called bean sprouts, as shown in Figure 1. In Thailand the bean sprouts are a basic food, appearing in dishes such as noodles with bean sprouts, stir fried bean sprouts with oyster, and stir fried bean sprouts with fried garlic as shown in Figure 2. It is a plant which readily germinates in normal soil or tissue paper on Earth. The previous study found that crew members aboard the International Space Station have been growing Mizuna Lettuce for years in their space station, confirming that astronauts are able to grow and safely eat space-grown vegetables as shown in Figure 3. However,
Lunar and Martian explorations have determined the mineral composition of the soils of these solar objects. Moreover, the rocks contain large amounts of sand-like soil or regolith. All essential minerals for the growth of plants appear to be present in sufficient quantities in both soils, probably with the exception of reactive nitrogen [2]. Charles C. Baskin investigated the relationship between seed density/specific gravity, seed quality, and plant performance. Generally, the high density seeds are of better quality than low density seeds. Germination increases as density/specific gravity increases [3]. Wuttichai S. and Sompong T. studied the effect of seed trimming and the development stage of the embryo for in vitro culture on seedling germination in rubber trees. The germination of the embryo was related to the water absorption and the enzyme activation process and the growth process of the seedlings. An embryo with cotyledon and without endosperm for in vitro culture can absorb water quickly and germimate instantly. On the other hand, the enzyme activation appears in the embryo with cotyledon and endosperm causing germination slower than in the former case [4]. 


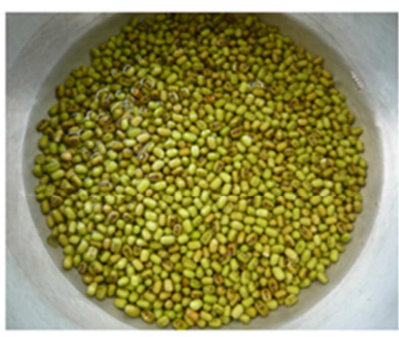

(a)

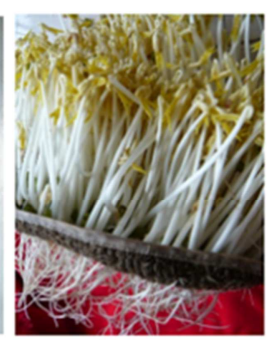

(b)
Figure 1. (a) The green bean seeds and (b) The bean sprouts.

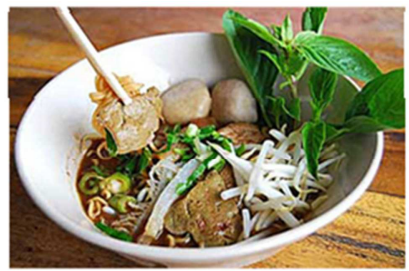

(a)

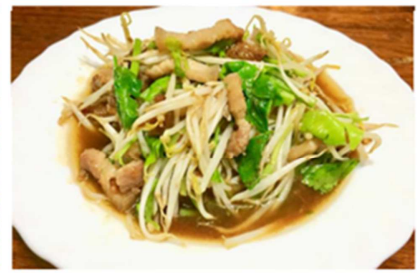

(b)
Figure 2. (a) Bean sprouts with noodles and (b) Fried bean sprouts.

It is known that gravity is dependent on the size of the planet: the moon has $1.62 \mathrm{~m} / \mathrm{s}^{2}$ and Mars has $3.711 \mathrm{~m} / \mathrm{s}^{2}$. Both are much less than the $9.81 \mathrm{~m} / \mathrm{s}^{2}$ on Earth. However, NASA has found new evidence of 219 planets outside our Solar System. Ten of those exoplanets appear to be similar to the size of the Earth and orbit their stars in the habitable zone. However, if a new Earth were the same size as Jupiter, then would plants be able to germinate and grow or not? In this study we want to answer this question by making use of pseudo gravity.

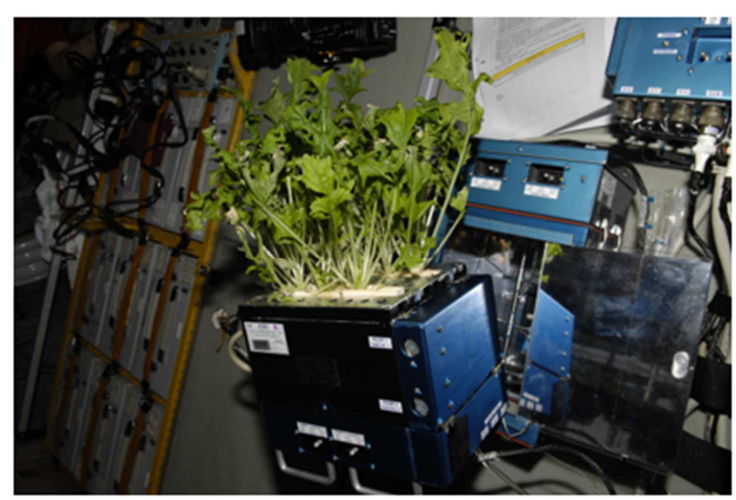

Figure 3. The lettuce growing on board the International Space Station. Image credit: NASA.

\section{Materials and Methods}

Imagine a moving object that can be modeled as a particle as shown in Figure 4. The force acting on a particle during centrifugation is given by the following equation [5]:

$$
F=m a_{c}
$$

Equation 1 can be written in another form

$$
F=m r \omega^{2}
$$

\section{Where}

$\mathrm{F}=$ The force acting on a particle during centrifugation

$r=$ The distance of the particle from the axis of rotation

$\omega=$ Angular velocity of the spinning rotor in radians per second

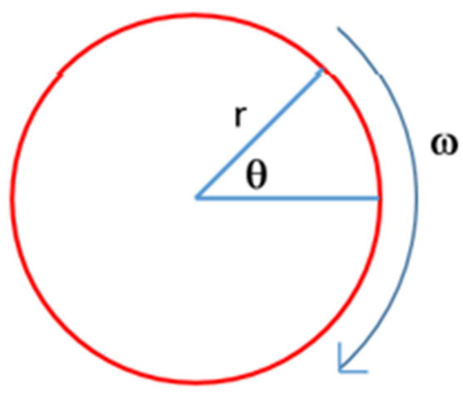

Figure 4. The circular motion.

During circular motion the acceleration is the product of the radius and the square of the angular velocity $\omega$, and the acceleration relative to "g". To find the Relative Centrifugal Force $(\mathrm{RCF})$ the mathematical description can be written in the form:

$$
R C F=\frac{F_{c e n}}{F_{g r a}}
$$

Substitute Equation 2 into Equation 3

$$
R C F=\frac{m r \omega^{2}}{m g}
$$

Finally,

$$
R C F=\frac{r \omega^{2}}{g}
$$

Because gravity is a constant, in this experiment $\mathrm{g}=980$ $\mathrm{cm} / \mathrm{s}^{2}$. In practice, the RCF has revolutions per minute (rpm) units. Where.

$$
\omega=\frac{d t}{d \theta}=\frac{2 \pi}{60} \mathrm{rpm}
$$

Thus Equation 5 can be written in the practical form:

$$
R C F=\frac{r\left(\frac{2 \pi}{60} r p m\right)^{2}}{980}
$$

Yielding

$$
R C F=r 1.119 \times 10^{-5}(r p m)^{2}
$$

Where $r$ is measured in centimetres.

Based on Equation 8 the centrifuge machine has been built with $r=24 \mathrm{~cm}$ as shown in Figure 5. The relationship between gravity and rpm is shown in Table 1 . 


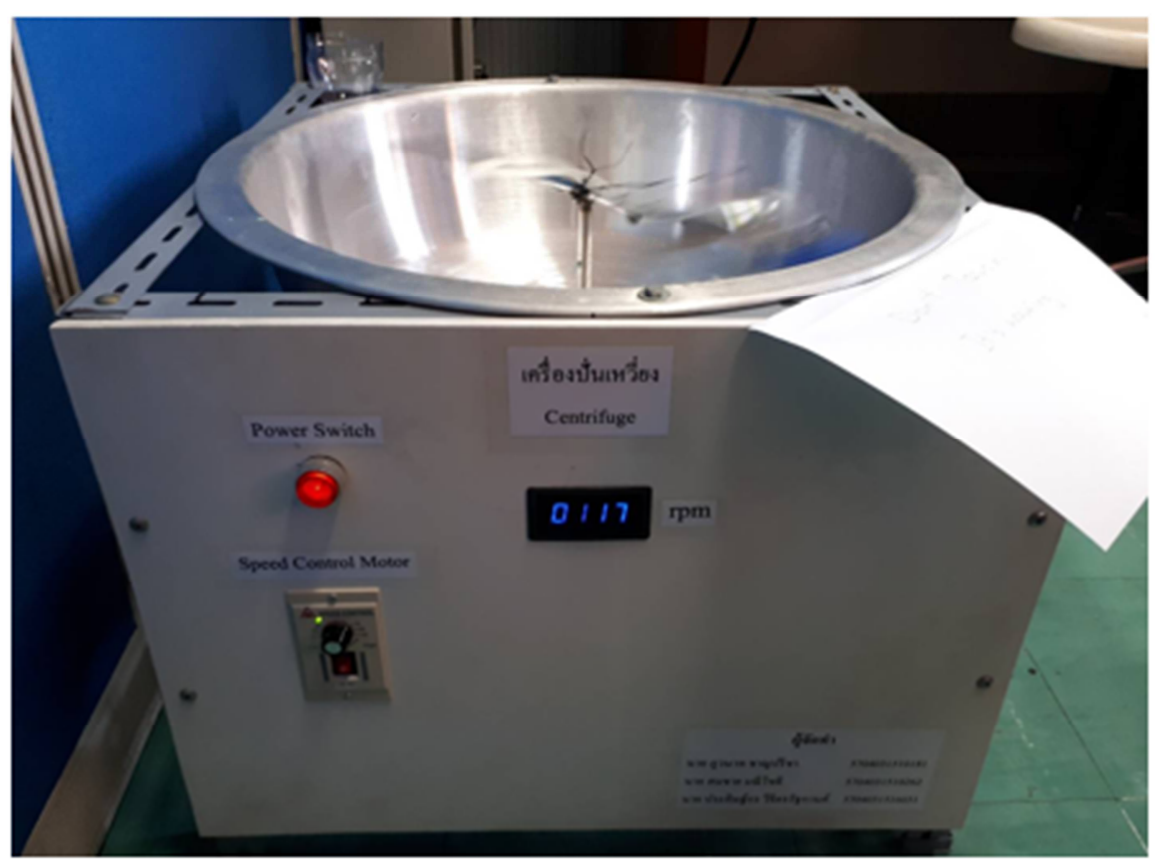

Figure 5. The centrifuge used in this research.

Table 1. The relationship between gravity and rpm.

\begin{tabular}{ll}
\hline G-Force & rpm \\
\hline 0.5 & 43.2 \\
1 & 61.4 \\
2 & 86.3 \\
2.5 & 96.5 \\
3 & 105.7 \\
3.5 & 114.2 \\
\hline
\end{tabular}

\section{Results}

The experiments were conducted using the centrifuge machine with five green bean seeds per case, with each case running 3 times. The results are shown in Table 2 .

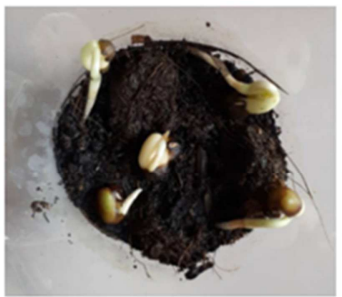

(a)

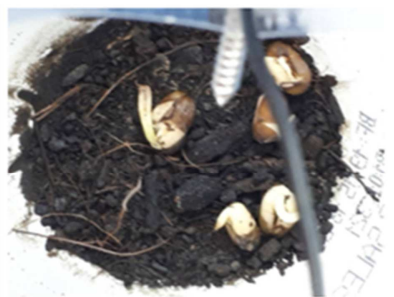

(b)
Figure 6. The behavior of germination and growth of green bean seeds at gravity of (a) $2 G$ and (b) $3 G$.

Table 2. The results of germination and growth.

\begin{tabular}{llll}
\hline G-Force & rpm & Germination & Growth \\
\hline 0.5 & 43.17 & yes & yes \\
1 & 61.4 & yes & yes \\
2 & 86.3 & yes & yes \\
2.5 & 96.5 & yes & no \\
3 & 105.7 & no & no \\
3.5 & 114.2 & no & no \\
\hline
\end{tabular}

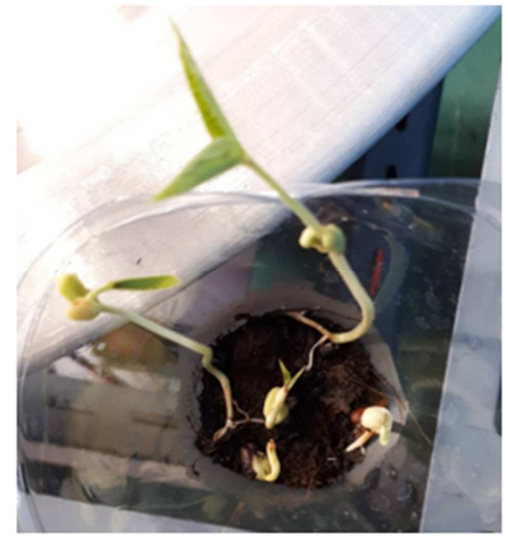

Figure 7. Bean sprouts.

Figure 6 (a) and Figure 7 show the results at $2 \mathrm{G}$ for the green bean seeds which were able to germinate and grow. Figure 6(b) reveals the behavior of the green bean seeds at $3 \mathrm{G}$ for which they were not able to grow but could germinate. Table 2 shows the capability of germination and growth at various gravity levels. The finite element modeling study by viscoelastic behavior [6]-[7] found that the maximum Von-Mises stress was on the stem of the bean sprout as shown in Figure 8. However, when the gravity was increased the stress was doubled on the stem. The cell walls in plants are composed of four basic building blocks: cellulose (the main structural fibre of the plant kingdom), hemicellulose, lignin, and pectin. Although the microstructure of plant cell walls varies in different types of plants, broadly speaking the cellulose fibres reinforce a matrix of hemicellulose and either pectin or lignin [8]. All portions have to resist the high gravity and are represented by viscoplastic behavior. 


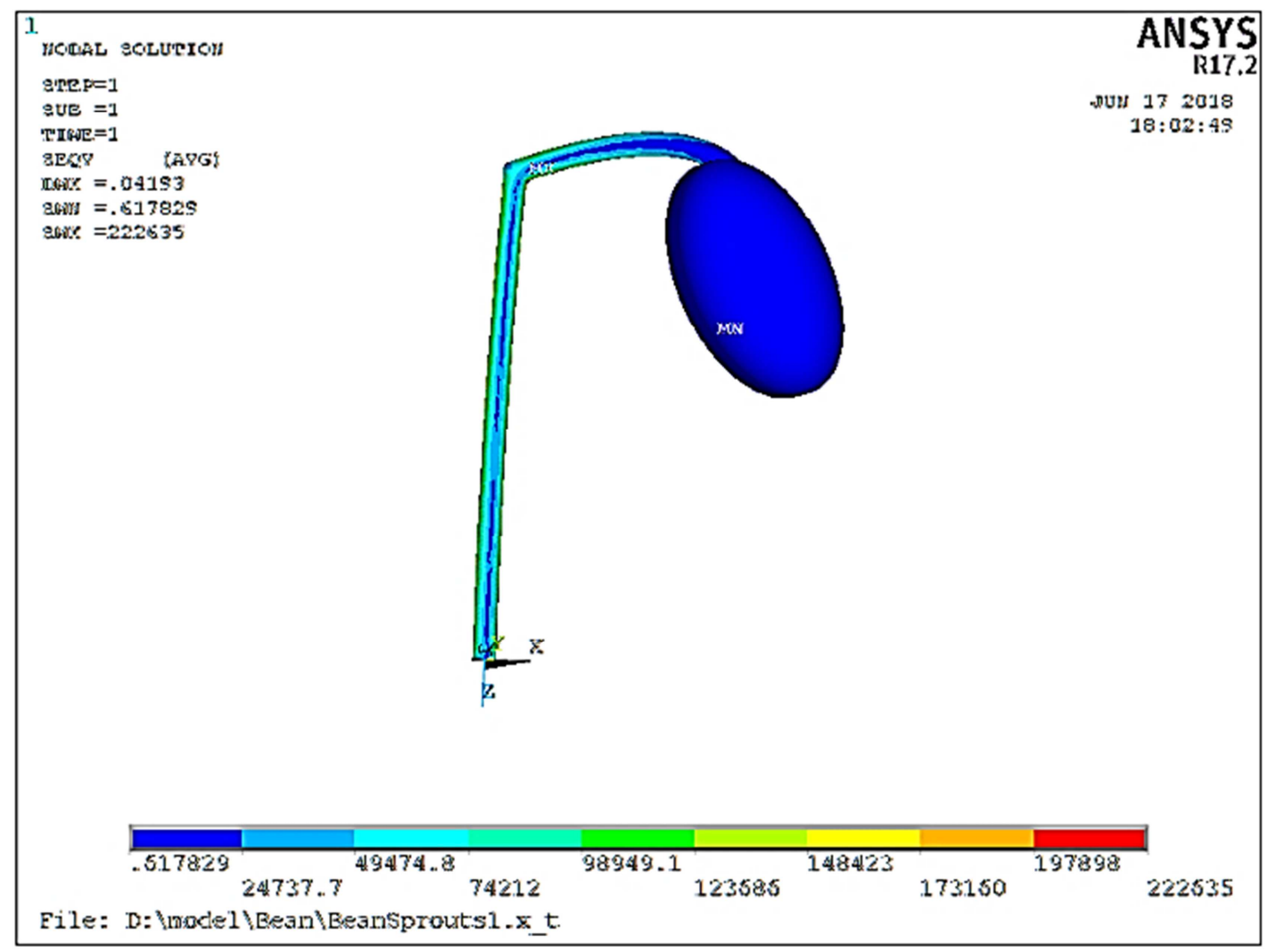

Figure 8. Von-Mises stress on the stem of the bean sprouts.

Nevertheless, this paper has been researched on Earth using gravity applied in the horizontal direction. Actually, the gravity on Earth acts in the vertical direction. Thus, the results of the resulting vector of the gravity takes the angle from the horizon line as shown in Figure 9. Also, the resulting vector of the gravity is the germination direction of the green beans.

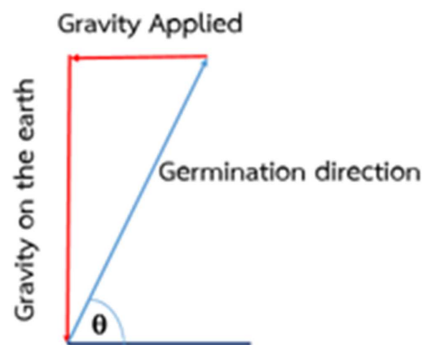

Figure 9. Germination direction of the green beans.

\section{Conclusion}

This investigation revealed that the plants will exhibit radial growth from the center of gravity, but it is true that the plant will sprout perpendicular to the surface of the planet. In this experiment, at the gravity was lower than $1 \mathrm{G}\left(9.81 \mathrm{~m} / \mathrm{s}^{2}\right)$ the plants were able to germinate and grow normally. However, when the gravity was greater than $2.5 \mathrm{G}$ the plants were able to germinate but not able to grow because the cell walls in the plant were not strong enough for the higher gravity. It might not be able to resist the high gravity, and thus the plants could grow on Mars and the moon but not on Jupiter at $2.5 \mathrm{G}$. It should be noted that due to the limitations of the authors' apparatus (speed control at low speed and the effect of the Earth's gravity), it was not possible to study the behavior of green beans at lower levels of gravity as the diameter was not large enough. The results from finite element analysis showed correlation with the experiment but did not take the angle from the horizontal line as actual germination. The findings appear reasonable. FEA also showed that at high levels of gravity the stems of the bean sprouts were not able to resist the force of gravity.

\section{Discussion}

In future works, the authors will improve the arm of the centrifuge and speed control, while the influence of gravity in the vertical plane perpendicular to the surface of the Earth can be reduced by adjusting the machine movement in the vertical instead of horizontal direction. Thus, the study of the growth of the green beans after the apparatus is improved might yield improved results. It might be possible to introduce bracing using small wood pieces beside the stem.

\section{Acknowledgements}

We would like to thank the Department of Industrial Physics 
and Medical Instrumentation, Faculty of Applied Science, King Mongkut's University of Technology North Bangkok for supporting every aspect of this research.

\section{References}

[1] Lori Meggs. Growing Plants and Vegetables in a Space Garden. [Online]. Available from: https://www. nasa.gov/ mission_pages/station/research/10-074.html. Retrieve Date: 4 March 2018.

[2] Wamelink GWW, Frissel JY, Krijnen WHJ, Verwoert MR, Goedhart PW (2014). Can Plants Grow on Mars and the Moon. [Online]. Available from: journals.plos.org/plosone/article?id=10.1371/journal.pone. 010 3138. Retrieve Date: 4 Jan 2018.

[3] Charles C. Baskin. The Relationship Between Seed Density/Specific Gravity, Seed Quality and Plant Performance. Mississippi State University. pp.67-81.

[4] Wutthichai S. and Sompong T. The Effect of Seed Trimming And Development Stage Of Embryo In Invitro Culture On Seedling Germination In Rubber Tree. Wicha Journal. Vol.33 No.1. January-June 2014.

[5] Raymond A. Serway and John W. Jewett, Jr. Physics for Scientists and Engineers with Modern Physics $9^{\text {th }}$ ed, Cengage Learning, 2014

[6] AVCalc LCC. (2018). [Online]. Available from: https: //www.aqua-calc.com/page/density-table/substance/mungblank-beans-coma-and-blank-mature-blank-seeds-coma-andblank-sprouted-coma-and-blank-raw
[7] E. Cakir, F. Alayunt and K. Ozden, 2002. A Study on the Determination of Poisson's Ratio and Modulus of Elasticity of Some Onion Varieties. Asian Journal of Plant Sciences, 1: 376-378.

[8] Lorna J. Gibson. The Hierarchical Structure and Mechanics Of Plant Materials. [Online]. Available from: https: //www.ncbi.nlm.nih.gov/pubmed/22874093. Retrieve Date: 4 March 2018.

[9] The Conversation Academic Rigour. Taking plants off planet how do they grow in zero gravity? [Online]. Available from: http: //theconversation.com/taking-plants-off-planet-how-dothey-grow-in-zero-gravity-45032. Retrieve Date: 2 February 2019.

[10] David Halliday, Robert Resnick, Jearl Walker. Fundamentals of physics 10th. John Wiley and Sons Inc, New York 2013.

[11] Erdogan Madenci, Ibrahim Guven. The Finite Element Method and Applications in Engineering Using ANSYS $2^{\text {nd }}$ ed, Springer, New York 2015.

[12] Plant in space. [Online]. Available from: https://en.wikipedia.org/wiki/Plants_in_space.

[13] Saeed Moaveni. Finite Element Analysis Theory and Application with ANSYS 1st ed, Pearson 1999.

[14] Kent L. Lawrence. ANSYS Tutorial Release 7.0, SDC Publications 2002

[15] James Owen. For National Geographic News. [Online]. Available from: https://news.nationalgeographic.com/news/2012/121207plants-grow-space-station-science/. Retrieve Date: 7 December 2012. 